\title{
Quality of Life of Older People with Dementia in Thailand
}

\author{
Linchong POTHIBAN ${ }^{1, *}$, Rojanee CHINTANAWAT ${ }^{1}$, \\ Nahathai WONGPAKARAN ${ }^{2}$, Chomphoonut SRIRAT ${ }^{1}$ and \\ Khanokporn SUCAMWANG ${ }^{1}$ \\ ${ }^{1}$ Faculty of Nursing, Chiang Mai University, Chiang Mai 50200, Thailand \\ ${ }^{2}$ Faculty of Medicine, Chiang Mai University, Chiang Mai 50200, Thailand
}

("Corresponding author's e-mail: linchong.p@cmu.ac.th)

Received: 8 March 2018, Revised: 6 January 2019, Accepted: 4 February 2019

\begin{abstract}
The quality of life (QOL) of older people with dementia may depend on their care environment. This cross-sectional descriptive study aims to investigate the QOL of Thai older people with dementia in homes for the aged and those living in their own homes in the community, as well as the discrepancy between the QOL rated by the older people and by caregivers. The samples included 342 participants who met the inclusion criteria. Data were collected using the Quality of Life-Alzheimer's Disease Scale (QOL-AD) and the EuroQol 5 Dimensions (EQ-5D) Questionnaire Thai Version Scale. Data were analyzed using descriptive statistics, t test, and Pearson's correlation. The findings revealed that the overall QOL scores of participants in both groups were at a moderate level. Compared with participants in homes for the aged, those living in the community showed higher scores in 7 aspects of QOL-AD, including physical health, energy, living situation, memory, self as a whole, ability to do chores, and ability to make life fun, but lower scores in the aspects of family/members and marriage/closed persons. Self-rated and caregiver-rated scores were significantly different in the aspects of living situation, memory, relationship with family, and marriage/closed persons. The findings imply that health care professionals can also assess the QOL of older people with dementia through self-rating. Further research to find the most effective method for enhancing older peoples' QOL is needed.
\end{abstract}

Keywords: Dementia, Quality of life, Older people, Homes for the aged, Caregiver-rated QOL

\section{Introduction}

It is estimated that there are now 46.8 million people living with dementia worldwide, with numbers projected to nearly double every 20 years, increasing to 74.7 million by 2030 , and 131.5 million by 2050 [1]. In Thailand, the prevalence of dementia from a national survey in 2014 was $8.1 \%$; prevalence of 8.7 $\%$ was reported in the northern region, $8.8 \%$ in the central region, $7.9 \%$ in the northeastern region, and $6.7 \%$ in the southern region [2]. This prevalence is expected to continuously increase due to the increased proportion of older population.

Dementia is a syndrome resulting from acquired brain disease and characterized by progressive deterioration in memory and other cognitive domains (e.g., language, judgment, abstract thinking, and executive functioning) [3]. Although the cognitive profiles of individuals diagnosed with dementia vary somewhat by etiology, the degree of deterioration represents a decline from previous levels of functioning and is sufficient to interfere with social and occupational functioning [4]. Additionally, personality changes and behavioral and psychological symptoms have often been reported [5]. A study in Thailand reported the top 5 neuropsychiatric symptoms of people with dementia to be nighttime motor behavior, depression, anxiety, irritability, and delusion [6]. Behavioral and psychological symptoms in dementia (BPSD) were found to be negatively associated with patient quality of life [7-10]. Moreover, at the late

Walailak J Sci \& Tech 2020; 17(10): 1066-1076. 
stage of the disease, when all memory is completely lost, patients may not be able to perform any activities of daily living and may even be unable to swallow [5]. They may become incontinent or at risk of malnutrition, pneumonia (especially due to aspiration), and pressure ulcers. Therefore, the quality of life (QOL) of people with dementia is probably decreased.

Because decline in cognitive and functional ability of people with dementia is progressive and incurable, maximizing their QOL has become the focus of dementia care and has been receiving more attention from health authorities and dementia researchers [11-12]. The World Health Organization defines QOL as "an individual perception of their position in life in the context of the culture and value systems in which they live and in relation to their goals, standards, and concerns" [13]. Currently, there is no consensus on a definition of QOL in dementia, but many researchers agree that QOL includes multiple domains of physical and mental health perceptions, among which mood and affect are critical components [14]. Therefore, many measures devised to assess QOL in dementia include mood, as well as other mental and physical aspects of life. Currently, many tools are available to measure health-related QOL that may be disease-specific or generic, depending on the QOL indicators. The EuroQoL 5 Dimensions Questionnaire (EQ-5D) is a well-developed generic measure that has been widely used in many countries by clinical researchers in a variety of clinical areas. With 5 questions for 5 dimensions of QOL, including mobility, self-care, usual activities, pain/discomfort, and anxiety/depression, EQ-5D has shown good characteristics of being relatively undemanding- taking a few minutes to complete- and seemingly being relevant to all respondents: healthy or severely ill; at home or in hospital; and of all ages [15]. Specific to dementia, the Quality of Life in Alzheimer's Disease Scale (QOL-AD), with 13 items [16], was found to be a well-known and effective measure of QOL that reflected the perceptions of individuals with dementia and their well-being [17]. However, it has been argued that a disease-specific measure is inadequate for describing and valuing QOL and, thus, a non-disease-specific instrument may be needed for complementing other measures [15]. Considering that both EQ-5D and QOL-AD are well developed and contain different aspects of QOL, they may be complementary when used in people with dementia.

Because QOL is a broad multidimensional concept that usually includes subjective evaluations of both positive and negative aspects of life, the assessment of each individual's own QOL should rely on self-perception, and patient rating may be the gold standard for measuring QOL in dementia. Previous studies have demonstrated that patients with mild to moderate dementia, or patients with Mini Mental State Examination (MMSE) scores of less than 10, can make reliable statements about their QOL [16,18], and other studies have reported that people with MMSE scores of 6 [9] and 3 [19] could rate their QOL. However, for people with dementia with cognitive impairments, and especially memory impairment, some researchers doubt that their self-reported QOL are reliable [20]. Therefore, all measures of QOL in dementia rely, at least partially, on caregiver or observer rating [14]. Previous studies in dementia have used patient rating, proxy rating, or both, and reported the discrepancies between proxy rating and patient rating. QOL has been consistently rated lower by caregivers than patient rating [9,21-24]. The lower ratings by caregivers were

associated with caregiver's burden [21] and caregiver's depression [23,25].

In Thailand, QOL in dementia is the major outcome of care, and needs more exploration. The majority of Thai older people with dementia are cared for in their own home by family members, particularly by children or a spouse [26], whereas some older people living at homes for the aged are cared for by the home's staff. In different living settings, the factors influencing QOL in dementia were found to differ [22]; therefore, QOL in dementia may be different for older people with dementia in different settings. Currently, comparative information is still lacking about the QOL of older people with dementia living in their homes versus those in the community, as well as about the ability of these older people to assess their own QOL. Also, due to the decreased ability of people with dementia to self-report their own QOL, patient-rated and caregiver-rated QOL of older people with dementia may be incongruent. As another requirement to account for cognitive impairment in dementia research subjects, the tools used for QOL assessment should be simple and reliable. Even though EQ-5D has been widely used in many populations, its applicability for Thai older people with dementia has not been reported. Therefore, there is still a need to understand the issues surrounding QOL assessment among Thai older 
people with dementia so that the data regarding the QOL of this particular group can be effectively used for planning further research and practices for preventing and delaying declines in their QOL.

The aims of the study are to describe the QOL levels of Thai older people with dementia using dementia-specific and generic measures, to compare the QOL of older people with dementia in homes for the aged with those living in the community, and to examine discrepancies in the QOL rated by older people and those rated by caregivers.

\section{Materials and methods}

\section{Research design and sample}

This study is part of a larger research investigating QOL and related factors in older people with dementia and caregivers, in which a cross-sectional descriptive design is used to examine the QOL of older people with dementia. The sample size was estimated for the correlational study, with five independent variables using Thorndike (1978)'s formula $(\mathrm{N}=10 \mathrm{~V}+50$, where $\mathrm{V}=$ number of variables) [27]. From calculation using $n=5$, the minimum number of a sample should be at least 100 . The researcher added $10 \%$ extra subjects to compensate for the possible incomplete responses; thereby, the sample size for older people with dementia was 110, and the number of caregivers was also 110 . However, the researcher decided to have an equal number (110) of older people from the 2 settings: homes for the aged and the community. Therefore, the planned number of samples were, in total, 330.

The samples of older people with dementia in homes for the aged and in the community were purposively selected based on the inclusion criteria. They were recruited if they had been diagnosed with dementia by health care professionals based on the Mini-Mental State Examination [MMSE]-Thai 2002 score (of $<22$ cut-off point in people who were educated at elementary school or higher, of 17 and lower in people who had education of lower than elementary school, and of 14 and lower in those who were illiterate [28]) and could verbally communicate.In total, there were 113 homes for the aged participants including 12 (10.62\%) from Baan Thammapakorn Social Welfare Development Center, Chiang Mai; 16 (14.16 \%) from Waithongniwes, Chiang Mai; 22 (19.47\%) from Lampang Social Welfare Development Center, Lampang; and 63 (55.75 \%) from Lopburi Social Welfare Development Center, Lopburi. The 110 community participants were selected from older people with dementia residing in Tak province and attending the Dementia Clinic of the Tak Provincial Hospital and its branches.

For caregivers, the participants were purposively selected from the care staff of homes for the aged and family caregivers who had taken care of older people with dementia for at least 3 months. There were 119 caregivers who participated in the study. Of this 119,43 were working at homes for the aged, and 76 were with dementia-affected older people in the community.

\section{Research instruments}

The three research instruments used to collect data were as follows:

1) The Quality of Life-Alzheimer's Disease (QOL-AD) questionnaire, originally devised by Logsdon et al. [16,23]. This is a 13-item questionnaire with 4 point scales, ranging from $1=$ poor to $4=$ excellent, used to obtain a rating of a patient's QOL from both the patients and the caregiver using indicators such as physical health, energy, mood, and living situation. The measure yields a single mean score ranging from 13 - 52, with higher mean scores indicating higher QOL. The QOL-AD was translated into Thai, with the permission of the Mapi Research Trust [29], using the back-translation technique. The original English version was translated into Thai by a bilingual expert, and then was translated back into English by two bilingual experts working independently. Discrepancies between the original version and the back-translated versions were checked and corrected until no discrepancies were found. The internal consistency reliability was tested with 10 Thai older persons, with the result of a Cronbach's alpha coefficient of 0.83 .

2) The EuroQoL 5 Dimensions Questionnaire (EQ-5D-3L) Thai Version, introduced in 1990 by the EuroQol Group [30]. This questionnaire was translated into Thai by Tongsiri [31]. The scale consisted of the index part, with 5 items measuring 5 dimensions of health-related QOL (HRQOL) including mobility, self-care, usual activities, pain/discomfort, and anxiety/depression, and the Visual Analog Scale part. 
Each dimension was rated for 3 levels of impairment, namely no problem (level 1, score $=1$ ), some/moderate problems (level 2, score $=2$ ), and extreme problems (level 3, score $=3$ ). The scores (utility scores) were calculated using a computer program [32]. The utility scores quantified HRQOL along a continuum that ranges from - 0.59 (worst health) to 1.00 (perfect health) [32]. The scores were classified into 3 levels with equal intervals [33]: -0.59-0.06 = low QOL, $0.07-0.60=$ moderate QOL, and $0.61-1.00=$ high QOL. In this study, the researcher had obtained permission to use this tool from the EuroQol Research Foundation, and used only the index part, because it was easier to be rated by older people with dementia. The internal consistency of this questionnaire was tested with 10 Thai older persons, with the result of a Cronbach's alpha coefficient of 0.92 .

3) A Demographic Data Recording Form, devised by the researcher to collect a range of demographic data about the older people with dementia and caregivers, including gender, age, marital status, education, income, comorbidity, smoking history, alcohol consumption, physical activity, and duration of having dementia.

\section{Procedure}

The study was approved by the research ethics committee, Faculty of Nursing, Chiang Mai University (reference number Full-029-2559). The heads of 4 government sponsored homes for the aged gave their permission to collect data from the older people with dementia in the home. All participants gave informed consent to take part in the study. Regarding dementia participants, written informed consent from their legal representatives was provided.

Before data collection, the researcher trained all collectors, who were doctoral students, by guiding them in how to ask the questions (items) in the instruments; then, the researchers and trained data collectors read all questions and answers to the participants item by item and marked the choices they selected. A sample of older people in homes for the aged and those dwelling in the community were asked to rate their own QOL using QOL-AD, whereas the caregivers in homes for the aged and those in the community were asked to rate the patients' QOL using EQ-5D-3L. To examine the discrepancies between self-rated QOL and caregiver-rated QOL, the caregivers in homes for the aged were also asked to rate the older peoples' QOL using QOL-AD. The data were collected from August, 2016, to January, 2017.

\section{Data analysis}

The data were analyzed using descriptive and inferential statistics. As data were normally distributed, an independent t-test was used to compare the QOL of older people with dementia between those in homes for the aged and in the community, and to compare self-rated and caregiver-rated QOL of dementia-affected older people. Pearson's correlation was used to examine the relationship between selfrated and caregiver-rated older peoples' QOL.

\section{Results and discussion}

\section{Sample characteristics}

Of 223 older people participants, $113(50.67 \%)$ were in homes for the aged and $110(49.32 \%)$ were living at home in the community. Most participants in homes for the aged and those in the community were female $(60.20$ vs $67.30 \%)$, aged 70 years and older $(78.80$ vs $76.40 \%)$, married $(50.40$ vs $44.50 \%)$, and had elementary school education (44.20 vs $73.6 \%$ ). Most participants were unemployed (89.40 vs $72.20 \%$ ) and had monthly income of less than 150 USD (5,000 THB) (97.30 vs 88.20\%) which was sufficient for their daily expenses. More than $75 \%$ of participants had comorbidities, which included hypertension (46.60 vs $52.73 \%$ ), diabetes (14.96 vs $9 \%$ ), dyslipidemia (27.30 vs $18.0 \%$ ), and previous stroke (10.60 vs $10.00 \%)$. More than $10 \%$ of participants were current smokers and $20-30 \%$ were exsmokers. About one third were extra-drinkers (34.40 vs $27.30 \%$ ), and almost half of participants (44.20 vs $48.20 \%$ ) were physically inactive. Regarding MMSE level [34], the MMSE scores in both groups were mostly at moderate. Those in homes for the aged had MMSE scores ranging from 4 to 21, with a mean of 13.49 (SD 5.38). Similarly, older people living in the community reported MMSE scores ranging 
http://wjst.wu.ac.th

from 4 to 20, with a mean of 13.50 (SD 4.08). The duration of having dementia ranged from 1 - 5 years, with a mean of 2.86 years, in homes for the aged group, and $1-14$ years, with a mean of 2.45 years, in the community group. The monthly income was $0-150$ USD $(5,000 \mathrm{THB})$, with a mean of 20.93 USD (697.58 THB), and $19-721$ USD (640 - 24,000 THB), with a mean of 76 USD (2,516 THB), in the homes for the aged group and community group, respectively.

Quality of life of older people with dementia measured by $Q O L-A D$

Both groups of older people participants were asked to rate their own QOL using the QOL-AD Scale $[14,22]$. The QOL scores ranged from 13 to 52, with a mean of 33.73 (SD 6.41), for those in homes for the aged, and from 13 to 43, with a mean of 30.50 (SD 6.20), for those in the community. The overall QOL scores for both groups were not significantly different (Table 1). When comparing the mean scores of each aspect of QOL-AD between the homes for the aged group and the community group, the data revealed that 9 items were significantly different $(p<0.01)$. The homes for the aged group showed significantly higher scores for the 7 aspects, including physical health, energy, living situation, memory, self as a whole, ability to do chores, and ability to make life fun $(p<0.01)$, but lower scores for the aspects of family/family member and marriage/closest friend $(\mathrm{p}<0.05)$ (Table 2$)$.

Table 1 QOL of older people with dementia measured by 2 instruments, comparing between those living in homes for the aged and those living in the community.

\begin{tabular}{lcccccc}
\hline \multicolumn{1}{c}{ QOL } & $\begin{array}{c}\text { Possible } \\
\text { score }\end{array}$ & $\begin{array}{c}\text { Homes for the aged } \\
(\mathbf{N = 1 1 3}) \\
\mathrm{M} \pm \mathrm{SD}\end{array}$ & Level & $\begin{array}{c}\text { Community } \\
(\mathbf{N}=\mathbf{1 1 0}) \\
\mathrm{M} \pm \mathrm{SD}\end{array}$ & Level & p value \\
\hline QOL-AD scores & $13-52$ & $33.73 \pm 6.41$ & Moderate & $30.50 \pm 6.20$ & Moderate & 0.97 \\
EQ-5D scores & $0-1$ & $0.56 \pm 0.38$ & Moderate & $0.51 \pm 0.38$ & Moderate & 0.71 \\
\hline
\end{tabular}

Table 2 Comparing aspects of QOL-AD among older people with dementia living in homes for the aged with those living in the community.

\begin{tabular}{lccccc}
\hline \multicolumn{1}{c}{ QOL-AD item } & $\begin{array}{c}\text { Possible } \\
\text { score }\end{array}$ & Range & $\begin{array}{c}\text { Homes for the aged } \\
(\mathbf{N = 1 1 3}) \\
\mathrm{M} \pm \mathrm{SD}\end{array}$ & $\begin{array}{c}\text { Community } \\
(\mathbf{N = 1 1 0}) \\
\mathrm{M} \pm \mathrm{SD}\end{array}$ & p value \\
\hline Overall & $13-52$ & $20-50$ & $33.73 \pm 6.41$ & $30.50 \pm 6.20$ & 0.97 \\
Physical health & $1-4$ & $1-4$ & $2.69 \pm 0.93$ & $2.00 \pm 0.80$ & 0.00 \\
Energy & $1-4$ & $1-4$ & $2.57 \pm 0.90$ & $1.69 \pm 0.79$ & 0.00 \\
Mood & $1-4$ & $1-4$ & $2.73 \pm 0.66$ & $2.55 \pm 0.82$ & 0.73 \\
Living situation & $1-4$ & $1-4$ & $3.17 \pm 0.72$ & $2.85 \pm 0.69$ & 0.00 \\
Memory & $1-4$ & $1-4$ & $2.58 \pm 0.82$ & $1.81 \pm 0.75$ & 0.00 \\
Relationship with family / family & $1-4$ & $1-4$ & $2.49 \pm 1.00$ & $2.79 \pm 0.72$ & 0.01 \\
member & & & & & \\
Marriage / closest persons / caregivers & $1-4$ & $1-4$ & $2.56 \pm 1.03$ & $2.81 \pm 0.68$ & 0.03 \\
Relationship with friends & $1-4$ & $1-4$ & $2.51 \pm 0.95$ & $2.45 \pm 0.63$ & 0.59 \\
Self as a whole & $1-4$ & $1-4$ & $2.75 \pm 0.81$ & $2.41 \pm 0.76$ & 0.00 \\
Ability to do chores & $1-4$ & $1-4$ & $2.37 \pm 0.98$ & $1.96 \pm 0.81$ & 0.00 \\
Ability to make life fun & $1-4$ & $1-4$ & $2.65 \pm 0.91$ & $2.26 \pm 0.74$ & 0.00 \\
Money/financial situation & $1-4$ & $1-4$ & $2.12 \pm 0.87$ & $2.18 \pm 0.67$ & 0.52 \\
Life as a whole & $1-4$ & $1-4$ & $2.55 \pm 0.93$ & $2.45 \pm 0.73$ & 0.40 \\
\hline
\end{tabular}


http://wjst.wu.ac.th

Quality of life of older people with dementia measured by EQ-5D-3L

The researcher firstly checked with the dementia participants as to whether or not they could answer the questions used in the EQ-5D-3L scale [30]. Most participants had difficulty answering the questions; therefore, rating by proxy was used. Regarding QOL level based on EQ-5D-3L scores, most of the homes for the aged participants reported no problems in all domains: mobility, self-care, usual activities, pain/discomfort, and anxiety/depression $(48.7,69.9,64.6,53.1$, and $60.2 \%$, respectively), while a lower percentage of community participants reported no problems in self-care and anxiety/depression (50.0 and $55.5 \%$, respectively), but a higher percentage reported moderate problems in mobility, usual activities, and pain/discomfort $(55.5,43.6$, and 59.1\%, respectively) (Table 3). The mean scores of QOL were 0.56 (SD 0.38) for the homes for the aged group, and 0.51 (SD 0.38) for the community group, which were not significantly different (Table 1), and were similarly classified as moderate level.

Table 3 Number and percentages of older people with dementia in homes for the aged and community based on QOL levels using EQ-5D (Caregiver-rated).

\begin{tabular}{lcccc}
\hline \multirow{2}{*}{ EQ-5D-3L domain / level } & \multicolumn{2}{c}{ Homes for the aged } & \multicolumn{2}{c}{ Community } \\
\cline { 2 - 5 } & $\mathbf{N}$ & $\mathbf{( \% )}$ & $\mathbf{N}$ & $\mathbf{( \% )}$ \\
\hline Mobility & & & & \\
$\quad$ No problem & 55 & $(48.67)$ & 39 & $(35.46)$ \\
Moderate problem & 42 & $(37.17)$ & 61 & $(55.45)$ \\
$\quad$ Extreme problem & 16 & $(14.16)$ & 10 & $(9.09)$ \\
$\quad$ Total & 113 & $(100.00)$ & 110 & $(100.00)$ \\
Self-care & & & & \\
$\quad$ No problem & 79 & $(69.91)$ & 55 & $(50.00)$ \\
Moderate problem & 19 & $(16.82)$ & 38 & $(34.55)$ \\
$\quad$ Extreme problem & 15 & $(13.27)$ & 17 & $(15.45)$ \\
Total & 113 & $(100.00)$ & 110 & $(100.00)$ \\
Usual activities & & & & \\
$\quad$ No problem & 73 & $(64.60)$ & 42 & $(38.18)$ \\
Moderate problem & 26 & $(23.01)$ & 48 & $(43.64)$ \\
$\quad$ Extreme problem & 14 & $(12.39)$ & 20 & $(18.18)$ \\
$\quad$ Total & 113 & $(100.00)$ & 110 & $(100.00)$ \\
Pain/discomfort & & & & \\
$\quad$ No problem & 60 & $(53.10)$ & 37 & $(33.64)$ \\
Moderate problem & 45 & $(39.82)$ & 65 & $(59.09)$ \\
$\quad$ Extreme problem & 8 & $(7.08)$ & 8 & $(7.27)$ \\
$\quad$ Total & 113 & $(100.00)$ & 110 & $(100.00)$ \\
Anxiety/depression & & & & \\
$\quad$ No problem & 68 & $(60.18)$ & 61 & $(55.45)$ \\
Moderate problem & 38 & $(33.63)$ & 46 & $(41.82)$ \\
$\quad$ Extreme problem & 7 & $(6.19)$ & 3 & $(2.73)$ \\
Total & 113 & $(100.00)$ & 110 & $(100.00)$ \\
\hline
\end{tabular}

The difference between self-rated and caregiver-rated quality of life of older people with dementia

To find the difference between self-rated and caregiver-rated QOL of older people with dementia, both older people with dementia and caregivers at homes for the aged were structurally interviewed using QOL-AD $[16,23]$. The findings revealed that self-rated QOL tends to be higher than caregiver-rated QOL. The overall QOL-AD scores from self-rating and caregiver-rating were not significantly different $(\mathrm{p}=$ 0.13). Of 13 aspects of QOL, only 4, living situation, memory, relationship with family/family member, 
http://wjst.wu.ac.th

and marriage/ closed persons/ caregivers demonstrated significantly higher self-rated scores than proxyrated $(\mathrm{p}<0.05)($ Table 4$)$.

The correlation between self-rated and caregiver-rated QOL of older people with dementia was also examined. The overall QOL-AD scores from self-rating and caregiver-rating were significantly positively correlated $(r=0.478, p<0.05)$. Significant correlations were found for all aspects, with the lowest $r$ of 0.201 for "relationship with friend," and the highest $r$ of 0.504 for "self as a whole" (Table 5).

Table 4 Comparing QOL-AD of older people with dementia in the Homes for the Aged between Self rated and Caregiver rated $(\mathrm{N}=113)$.

\begin{tabular}{|c|c|c|c|c|}
\hline & QOL item & $\begin{array}{c}\text { Self- rated } \\
\mathrm{M} \pm \mathrm{SD}\end{array}$ & $\begin{array}{c}\text { Caregiver- rated } \\
\mathrm{M} \pm \mathrm{SD}\end{array}$ & p value \\
\hline \multirow[t]{14}{*}{ Overall } & & $33.73 \pm 6.41$ & $32.18 \pm 6.70$ & 0.13 \\
\hline & Health status & $2.69 \pm 0.93$ & $2.61 \pm 0.87$ & 0.37 \\
\hline & Energy & $2.57 \pm 0.90$ & $2.47 \pm 0.88$ & 0.32 \\
\hline & Mood & $2.73 \pm 0.83$ & $2.68 \pm 0.75$ & 0.56 \\
\hline & Living situation & $3.17 \pm 0.72$ & $2.93 \pm 0.82$ & 0.05 \\
\hline & Memory & $2.58 \pm 0.82$ & $2.38 \pm 0.82$ & 0.02 \\
\hline & Relationship with family / family member & $2.49 \pm 1.00$ & $2.20 \pm 0.85$ & 0.01 \\
\hline & Marriage / closed persons / caregivers & $2.56 \pm 1.00$ & $2.29 \pm 0.82$ & 0.00 \\
\hline & Relationship with friends & $2.51 \pm 0.95$ & $2.45 \pm 0.74$ & 0.54 \\
\hline & Self as a whole & $2.75 \pm 0.81$ & $2.66 \pm 0.65$ & 0.21 \\
\hline & Ability to do chores & $2.27 \pm 0.98$ & $2.20 \pm 0.93$ & 0.06 \\
\hline & Ability to make life fun & $2.65 \pm 0.91$ & $2.57 \pm 0.79$ & 0.33 \\
\hline & Money/financial situation & $2.12 \pm 0.87$ & $2.28 \pm 0.83$ & 0.06 \\
\hline & Life as a whole & $2.55 \pm 0.93$ & $2.46 \pm 0.67$ & 0.31 \\
\hline
\end{tabular}

Table 5 Correlation between Self-rated QOL and Caregiver- rated QOL.

\begin{tabular}{lcc}
\hline \multicolumn{1}{c}{ QOL } & r & p value \\
\hline Overall & 0.478 & 0.000 \\
Health status & 0.375 & 0.000 \\
Energy & 0.318 & 0.001 \\
Mood & 0.267 & 0.004 \\
Living situation & 0.354 & 0.000 \\
Memory & 0.425 & 0.000 \\
Relationship with family/family member & 0.377 & 0.000 \\
Marriage / closed persons/caregivers & 0.473 & 0.000 \\
Relationship with friends & 0.201 & 0.033 \\
Self as a whole & 0.504 & 0.000 \\
Ability to do chores & 0.474 & 0.000 \\
Ability to make life fun & 0.495 & 0.000 \\
Money/financial situation & 0.411 & 0.000 \\
Life as a whole & 0.382 & 0.000 \\
\hline
\end{tabular}




\section{Discussion}

This study compared the QOL of Thai older people with dementia living in government sponsored homes for the aged with that of people living in their own homes in the community. QOL was assessed using both the QOL-AD, original version, and the EQ-5D, the "3L" version. Older people with dementia in both homes for the aged and in the community completed the QOL-AD Scale by themselves, while their caregivers rated their QOL using both the QOL-AD and the EQ-5D-3L. As demonstrated in some research, in which patients with MMSE scores of 10 or higher can usually complete the questionnaire without problems $[16,18,35]$, in this study, the older people who could rate their QOL had an even lower MMSE score of 5 (7.1\% in government sponsored homes for the aged, and $7.3 \%$ in the community). Two previous studies also reported successful use of the QOL-AD with persons with MMSE scores as low as 3 [17,25]. In this study caregivers also could use it without difficulty. Therefore, QOL-AD with 13 items can be used with Thai older people with dementia and caregivers.

The findings revealed that the QOL of older people with dementia in homes for the aged and those living in the community, as measured by the 2 different instruments, when classified into levels of low, moderate, and high by the possible scores, was at a moderate level. The majority of older people with dementia in both groups had no problems in their health-related QOL. Even though the older people with dementia dwelling in the community tended to show lower QOL than those in homes for the aged, the difference was not statistically significant. In this study, older people with mild to moderate dementia were included. Not many older people had severe behavioral or psychological symptoms, the factor negatively related to patients' QOL [7-10]. This may explain moderate QOL in older people with dementia.

The similarity of older peoples' QOL in both groups may be due to the similarity in caring activities provided to them by caregivers. In the current study, older people with dementia in government sponsored homes for the aged are cared for by caregivers under the supervision of a nurse (if available), while those dwelling in the community are cared for by family caregivers. In Thai society, long term care for dependent older people, including those with dementia, is mostly $(95.5 \%)$ provided by a family member, particularly the daughter or spouse, and $9.5 \%$ is provided by paid caregivers [36]. Currently, family caregivers do not have any formal training and lack knowledge and skills regarding dementia care. Almost all caregivers said they knew very little about dementia or care. They may be able to assist older people with dementia in activities of daily living but may not be able to properly respond to their diseaserelated needs and problems. Similarly, caregivers in government-sponsored homes for the aged in Thailand, with limited formal education, may have on the job-training in basic care for older people, but very little, or none, specifically on dementia care. However, in some government-sponsored homes for the aged, there may be some regular services from health care professionals and students, particularly psychiatrists or general practitioners, available for the residents. This may explain why dementia patients in homes for the aged were more satisfied with almost all aspects of their life than were those in the community. In this study, people residing in homes for the aged had significantly higher satisfaction with health status, energy, living situation, memory, self as a whole, ability to do chores, and ability to make life fun. The better feeling towards those aspects of life may result from the services received and daily activities arranged for them by all homes for the aged, including medical services, recreational activities, exercise, and religious practices. In this study, one home for the aged even had weekly mental health care service for the residents. On the other hand, most older people with dementia dwelling in the community were kept at home and separated from social activities because of dementia. They were taken care of only by family members; therefore, they were more satisfied with family and marriage/closest persons than were older people in homes for the aged.

When comparing the QOL of older people with dementia as rated by older people themselves and by caregivers, this study demonstrated no significant difference, even though the self-rated QOL scores were a little higher than the caregivers' rated scores. These findings are not consistent with previous studies, which showed discrepancies between patient-rated and caregiver-rated QOL of dementia-affected older people, where people with dementia provided higher ratings [9,37-39]. The similar views on QOL of older people with dementia of the 2 raters may be due to the closeness and good relationship between older people and caregivers in homes for the aged in Thailand. The caregivers generally know how older 
http://wjst.wu.ac.th

people feel and surprisingly very often can promptly respond to the older peoples' needs even before the older people make requests.

\section{Limitations}

The data collection was conducted through the self-reporting of the samples. The information may be incorrect, despite our best efforts to be honest and accurate. This could be noted as a limitation. Furthermore, most of the participants were female; therefore, the results might not be generalized to the male population. Further study to compare between male and female older persons with dementia is required.

\section{Implications of the study}

The results of this study provide health care providers working with dementia-affected patients with information regarding QOL assessment, including both tools and methods. To assess patient QOL, input from the patients themselves is necessary. Having older people with dementia rating their own QOL is possible, even in moderate dementia. Rating by proxy, when necessary, is good enough for assessing older peoples' QOL. The findings also provide health care leaders with the evidence to improve the QOL of Thai older people with dementia in both institutes and the community.

\section{Conclusions}

The QOL of both Thai older people with mild to moderate dementia living in homes for the aged and those living in their own homes in the community can be assessed using different tools by self-rating and proxy-rating. QOL-AD is easier than EQ-5D for self-rating in older people. To use the QOL-AD questionnaire with older people with dementia in homes for the aged, the researcher may have to adapt some items to fit the group; for example, items regarding family might be adapted to those living together with the aged in the home. Study findings are information for health care professionals pertaining to QOL assessment and maintenance in older people with dementia.

\section{Acknowledgements}

This paper presents, in part, the results of a broader research entitled "Quality of Life of Older People with Dementia and Caregivers", granted by Chiang Mai university, Thailand. The authors gratefully acknowledge the great assistance in data collection from Ms. Janya Jainoon, RN, MSN, at Somdejphrajaotaksin Maharaj Hospital, Tak province; Ms. Rattanaporn Wattana, RN, at Lopburi Home for the Aged, and Pikul Uthaya, Phanit Leejaroern, and other doctoral students in the Ph.D program at Chiang Mai University, Faculty of Nursing.

\section{References}

[1] World Alzheimer Report 2015. Available at: https://www.asz.co.uk, accessed March 2017.

[2] W Ekpalakorn. Demographic, Social, and Economic Characteristics. In: W Ekpalakorn (Ed.). Report: The survey of Thai health people by physical examination 2014. $5^{\text {th }}$ ed. Health System Research Institute, Nontaburi, 2014, p. 1-253.

[3] M Bourgeois and E Hickey. Dementia: From Diagnosis to Management: A Functional Approach. Psychology Press, New York, 2009.

[4] The American Speech-Language-Hearing Association, ASHA. Available at: https://www.asha.org/ public/speech/disorders/dementia, accessed March 2017.

[5] J Huang. Overview of Delirium and Dementia, Available at: http://www.msdmanuals.com/ professional/neurologic-disorders/delirium-and-dementia/overview-of-delirium-and-dementia, accessed January 2017.

[6] S Veragiat, WC Pensuksan, KS Suvanchot, C Yooyen, P Chetkhunthod and P Sarsanas. Prevalence of dementia and neuropsychiatric symptoms among elderly patients attending outpatient departments of psychiatric hospitals in southern Thailand. Walailak J. Sci. \& Tech. 2016; 14, 43-9. 
http://wjst.wu.ac.th

[7] S Banerjee, SC Smith, DL Lamping, RH Harwood, B Foley, P Smith, J Murray, M Prince, E Levin, A Mann and M Knapp. Quality of life in dementia: More than just cognition. An analysis of associations with quality of life in dementia. J. Neurol. Neurosurg. Psychiatr. 2006; 77, 146-8.

[8] A Burns. The burden of Alzheimer's disease. Int. J. Neuropsychopharmacol. 2000; 3, 31-8.

[9] C Hurt, S Bhattacharyya, A Burns, V Camus, R Liperoti, A Marriott, F Nobili, P Robert, M Tsolaki, B Vellas, F Verhey and EJ Byrne. Patient and caregiver perspectives of quality of life in dementia. Dement. Geriatr. Cognit. Disord. 2008; 26, 138-46.

[10] T Matsui, S Nakaaki, Y Murata, J Sato, Y Shinagawa, H Tatsumi and TA Furukawa. Determinants of the quality of life in Alzheimer's disease patients as assessed by the Japanese version of the quality of life: Alzheimer's Disease Scale. Dement. Geriatr. Cogn. Disord. 2006; 21, 182-91.

[11] E Moniz-Cook, M Vernooij-Dassen, R Woods, F Verhey, R Chattat, MD Vugt, G Mountain, M O'Connell, J Harrison, E Vasse, RM Dröes and M Orrell. A European consensus on outcome measures for psychosocial intervention research in dementia care. Aging Ment. Health 2008; 12, 1429.

[12] P Raeymaekers and D Roger. Improving the Quality of Life of People with Dementia in the EU: A Challenge for the European Society. King Baudouin Foundation, Brussels, 2010.

[13] WHO QOL Group. The World Health Organization quality of life assessment (WHOQOL): Position paper from the World Health Organization. Soc. Sci. Med. 1995; 41, 1403-9.

[14] RE Ready. Quality of life in dementia. Med. Health Rhode Island 2002; 85, 216-8.

[15] R Rabin and F de Charro. EQ-5D: A measure of health status from the EuroQol group. Ann. Med. $2001 ; 33,337-43$.

[16] RG Logsdon, LE Gibbons, SM McCurry and L Teri. Quality of life in Alzheimer's disease: Patient and caregiver reports. J. Ment. Health Aging 1999; 5, 21-32.

[17] J Hoe, G Hancock, G Livingston and M Orrell. Quality of life of people with dementia in residential care homes. Br. J. Psychiatr. 2006; 188, 460-4.

[18] CG Mozley, P Huxley, C Sutcliffe, H Bagley, A Burns, D Challis and L Cordingley. 'Not knowing where I am doesn't mean I don't know what I like': Cognitive impairment and quality of life responses in elderly people. Int. J. Geriatr. Psychiatr. 1999; 14, 776-83.

[19] J Hoe, C Katona, B Roch and G Livingston. Use of the QOL-AD for measuring quality of life in people with severe dementia: The LASER-AD study. Age Ageing 2005; 34, 130-5.

[20] PV Rabins and BS Black. Measuring quality of life in dementia: purposes, goals, challenges and progress. Int. Psychogeriatr. 2007; 19, 401-7.

[21] M Crespo, MB de Quirós, MM Gómez and C Hornillos. Quality of life of nursing home residents with dementia: A comparison of perspectives of residents, family, and staff. Gerontologist 2012; 52, 56-65.

[22] W Jing, R Willis and Z Feng. Factors influencing quality of life of elderly people with dementia and care implications: A systematic review. Arch. Gerontol. Geriatr. 2016; 66, 23-41.

[23] RG Logsdon, LE Gibbons, SM McCurry and L Teri. Assessing quality of life in older adults with cognitive impairment. Psychosom. Med. 2002; 64, 510-9.

[24] CE Selai, MR Trimble, MN Rossor and RJ Harvey. Assessing quality of life in dementia: Preliminary psychometric testing of the Quality of Life Assessment Schedule (QOLAS). Neuropsychol. Rehabi. 2001; 11, 219-243.

[25] A Selwood, A Spector, L Royan, M de Madariaga Lopez, R T Woods and M Orrell. Whose quality of life is it anyway? The validity and reliability of the Quality of Life-Alzheimer's Disease (QoLAD) scale. Alzheimer Dis Assoc. Disord. 2003; 17, 201-8.

[26] Foundation of Thai Gerontology Research and Development Institute. Situation of the Thai elderly 2015, Available at: http://www. thaitgri.org, accessed January 2017.

[27] RM Thorndike. Correlation Procedures for Research. Gardner Press, New York, 1978.

[28] Thai Cognitive Test Development Committee 1999. Mini-Mental State Examination-Thai 2002 (in Thai). Ministry of Public Health, Bangkok, 2002. 
[29] Mapi Research Trust. Quality of life in Alzheimer's disease (QOL-AD), Available at: https://eprovide.mapi-trust.org/instruments/quality-of-life-in-alzheimer-s-disease, accessed February 2017.

[30] EuroQol Group. EuroQol-a new facility for the measurement of health-related quality of life. Health Policy 1990; 16, 199-208.

[31] S Tongsiri. The Thai Population-based Preference Scores for EQ-5D Health States (in Thai). Ministry of Public Health, Nonthaburi, 2009.

[32] BV Hout, MF Janssen, YS Feng, T Kohlmann, J Busschbach, Dominik Golicki, Andrew Lloyd, Luciana Scalone, Paul Kind, A Simon Pickard. Interim scoring for the EQ-5D-5L: Mapping the EQ5D-5L to EQ-5D-3L value sets. Value Health 2012; 15, 708-15.

[33] K Vanichbuncha. Principle of statistics (in Thai). $7^{\text {th }}$ ed. Chulalongkorn University, Bangkok,2004.

[34] MF Folstein, SE Folstein and, PR McHugh. Mini-mental state: A practical method for grading the cognitive state of patients for the clinician. J. Psychiatr. Res. 1975; 12, 189-98.

[35] A Selwood, L Thorgrimsen and M Orrell. Quality of life in dementia: A one year follow up study. Int. J. Geriatr. Psychiatr. 2005; 20, 232-7.

[36] S Srihapark, K Chuengsatiansup and K Tengrang. Impact and long term caregiving burden in Thai culture: A research report (in Thai). The Health System Research Institute, Bangkok, Available at: http://www.kb.hsri.or.th/dspace/handle/11228/4028?locale-attribute=th, accessed January 2017.

[37] S Arlt, J Hornung, M Eichenlaub, H Jahn, M Bullinger and C Petersen. The patient with dementia, the caregiver and the doctor: cognition, depression and quality of life from three perspectives. Int. J. Geriatr. Psychiatr. 2008; 23, 604-10.

[38] W Moyle, JE Murfield, SC Griffiths and L Venturato. Assessing quality of life of older people with dementia: A comparison of quantitative self-report and proxy accounts. J. Adv. Nurs. 2011; 68, 2237-46.

[39] A Vogel, EL Mortensen, SG Hasselbalch, BB Andersen and G Waldemar. Patient versus informant reported quality of life in the earliest phases of Alzheimer's disease. Int. J. Geriatr. Psychiatr. 2006; 21, 1132-8. 